\title{
Impacts of the Properties Heterogeneity on 3D Magnetic Dusty Nanofluids Flow Within Cubic Porous Enclosures Contain Isothermal Cylinders
}

\section{Z. Z. Rashed ( $\nabla$ zalanazi@ju.edu.sa ) Jouf University}

\section{Research Article}

Keywords: 3D flow, dusty nanofluid, non-homogeneous model, heterogeneous porous medium, isothermal cylinders

Posted Date: November 1st, 2021

DOI: https://doi.org/10.21203/rs.3.rs-967501/v1

License: (9) This work is licensed under a Creative Commons Attribution 4.0 International License.

Read Full License 


\title{
Impacts of the properties heterogeneity on 3D magnetic dusty nanofluids flow within cubic porous enclosures contain isothermal cylinders
}

\author{
Z. Z. Rashed \\ Mathematics Department, Faculty of Science and Arts, Jouf University, Qurayyat, Saudi \\ Arabia \\ Email: zalanazi@ju.edu.sa
}

\begin{abstract}
This paper examines the controlling of the three dimensional dusty nanofluid flow using the two circular cylinders having different thermal conditions. The cylinders are located in the middle area while the location of the right cylinder is changeable. The $3 \mathrm{D}$ cubic flow domain is filled by a non-Darcy porous medium and a magnetic field in Z-direction is taken place. The non-homogeneous two phase model of the nanofluid is applied while the permeability and thermal conductivity of the porous medium are assumed heterogonous. The current situation is represented by two systems of the equations for the nanofluid and dusty phases. The solutions methodology is depending on the 3D SIMPLE scheme together with the finite volume method. The major outcomes indicating to that the flow can be well controlled using the inner isothermal cylinders. Also, the cases of the heterogeneity in $X-Y$ and $X-Z$ directions give the lowest values of $N u_{a v}$.
\end{abstract}

Keywords: 3D flow, dusty nanofluid, non-homogeneous model, heterogeneous porous medium, isothermal cylinders.

\section{Introduction}

Examining of the natural convection flow within closed domains in porous media has received much attention from many researchers because of its widespread applications in various fields, such as engineering systems, electronics cooling, geothermal reservoirs, nuclear reactors, regenerative heat exchangers, electric machinery, solar collectors, air conditioning, and chemical industries. Such applications have been found in several studies, for example in [1-5]. On the other hand, nanofluids have gained great importance over the past years due to superior performance on the improvement of thermal conductivity 
compared to basic fluids. The nanofluid is defined as a fluid consists of a basic liquid mixture with solid nanoparticles (nanosized solid particles such as $\mathrm{Cu}, \mathrm{Ag}, \mathrm{CuO}$ and $\mathrm{Al}_{2} \mathrm{O}_{3}$ ) to the base fluid of dimensions less than $100 \mathrm{~nm}$. There are several published papers on dealt with thermophysical properties of nanoparticles, development and preparation can be found in [611]. Afterward, different numerically and experimentally studies for different surfaces and media were presented; for example but not limited to [12-19].

May by reviewing the existing literatures on the natural convection of nanofluids, it was found that few researchers have studied the nanoparticles in a 3D domains with/without porous enclosure. Jelodari, and Nikseresht [20] discussed the effect of the Lorentz force on the thermal performance of nanofluids in a cubic cavity. They used a numerical technique based on the finite volume method. They observed that the conduction heat transfer is predominated when the concentration of the nanoparticles is increased up to 6\%. Sajjadi et al. [21] investigated MHD natural convection in a cubic cavity with sinusoidal boundary conditions. They indicated to with the increase of the Rayleigh number and nanoparticles' volumetric fraction, the $\mathrm{Nu}$ is increased. Wang et al. [22] analyzed the natural convection of the nanofluids in a partially heated cubic enclosure. They found that if the aspect ratio increases, the average Nusselt number, and the heat transfer are decreased. Sheikholeslami et al. [23] discussed the lattice Boltzmann method for the roles of magnetic field on the free convection of the nanofluids in the porous media using the non-Darcy model. From the results, it is seen that the increased Darcy number leads to the temperature boundary layer thickness becomes thinner. Sheremet and Pop [24] investigated the natural convection in a heated cubical cavity under the Marangoni effect. They applied the finite difference method then discussed the impacts of the controlling parameters on the velocity, temperature, nanoparticles volume fraction and the average Nusselt number. Sheikholeslami et al. [25] considered a cubical enclosure in the existence of a magnetic field with hot sphere obstacle in the flow domain. They found that the increase of Darcy number leads to the thermal boundary layer becomes thicker. Alsabery et al. [26] discussed the unsteady 3D natural convection heat transfer inside a wavy porous cubical area using a Galerkin weighted residual scheme based on the finite element method. They observed that the increasing in Da causes that the Nusselt number is significantly increased.

On the other side, many researchers concentrated on the case of containing small solid particles like dust particles. This mixture type called dusty fluid. Study the properties of these types of liquids have wide range of applications such that, cooling systems, flows in rocket 
tubes, environmental pollutants and in engineering and sciences...etc. These applications can be found in Marble [27] and Rudinger [28]. Afterward several studies have been extended to the case of the dusty nanofluids. Naramgari and Sulochana [29] studied dusty the nanofluid flow over a stretching surface with effect of the magnetic force. Their governing system is solved numerically by using Runge -Kutta based shooting technique. They found that an increase in Hartmann number help to decrease the friction factor. Begum et al. [30] studied the problem of bioconvection boundary layer the flow of two-phase dusty nanofluids. They used numerical solutions and found that the rising in the buoyancy ratio and mass concentration parameters leads to a reduction in the skin friction coefficient. Siddiqa et al. [31] investigated the natural convection flow of two phase dusty nanofluid along a vertical wavy surface. From the results, the temperature profiles tend to increase with the growing in modified diffusivity ratio. Gireesha et al. [32] discussed the Hall current effect on the dusty nanofluid flow and used the Runge-Kutta-Fehlberg method coupled with shooting algorithm. Their results pointed to that the Hall effects lead to accelerate the velocities.

Mishra et al. [33] investigated the magnetic field effect on the dusty nanofluids in a porous medium. They interested in the influences of the governing parameters on the skin friction coefficient and the heat transfer rate for both the fluid and dusty phases. Rashid et al. [34] studied the mixed convection in a porous medium with radiation effect. They concluded that an increase of the volume fraction of the nanoparticles leads to an enhancement in the Nusselt coefficient. Recently, Rashed and Ahmed [35] discussed the peristaltic flow of a dusty nanofluid in curved channels.

Non-homogeneous model has been used by few researchers to study natural convection of the nanofluids saturated porous cubic cavities. Zhuang and Zhu [36-37] studied the buoyancy Marangoni convection of non-Newtonian nanofluids with a heterogeneous porous medium, numerically, by applying the finite volume method. They found that the level of heterogeneity controls the entropy generation and decreases the heat transfer rate. Rashed et al. [38] investigated the unsteady 3D nanofluid flow within a cubic enclosure filled with a heterogeneous porous medium and they discussed different cases.

As evident from the above literature review, no research yet dealing with the unsteady natural convection flow of dusty nanofluid flow within cubic enclosure in a porous medium with hot and cold cylinders. Hence, the aim of this study is investigating the dusty nanofluid flow within the cubic enclosure in a porous medium with hot and cold cylinders in the presence of 
the magnetic field and a heterogeneous porous medium influences. During this simulation, we discuss the effects different parameters of problem on the temperature, velocity for dusty /nanofluid phases and the average Nusselt number.

\section{Assumptions and analyses}

Consider a time-dependent and three dimensional flow within a porous cubic container in the presence of a magnetic field as depicted in Figure 1. The mixture is a nanofluid contains dusty particles while the domain contains two-cylinders. One of the cylinders is hot $\left(T=T_{h}\right)$ and the other is cold $\left(T=T_{c}\right)$ where $\left(T_{h} \gg T_{c}\right)$; those are spreated by a distance $\delta$. It is assumed, also, that the porous medium is non-homogeneous in all directions where the permeability and thermal conductivity are varied exponentially in $x-, y-$ and $z$-directions. Additionally, the magnetic field is taken in z-direction with constant strength $\beta_{0}$. Table 1 presents the thermophysical properties of the host fluid and nanoparticles. Furthermore, the non-homogeneous nanofluid model is applied to represent this physical case and two systems of equations are introduced as:

\section{Nanofluid phase:}

$\frac{\partial u}{\partial x}+\frac{\partial v}{\partial y}+\frac{\partial w}{\partial z}=0$

$\rho_{n f}\left[\frac{1}{\varepsilon} \frac{\partial u}{\partial t}+\frac{u}{\varepsilon^{2}} \frac{\partial u}{\partial x}+\frac{v}{\varepsilon^{2}} \frac{\partial u}{\partial y}+\frac{w}{\varepsilon^{2}} \frac{\partial u}{\partial z}\right]=-\frac{\partial p}{\partial x}+\frac{\mu_{n f}}{\varepsilon}\left(\frac{\partial^{2} u}{\partial x^{2}}+\frac{\partial^{2} u}{\partial y^{2}}+\frac{\partial^{2} u}{\partial z}\right)-\frac{\mu_{n f}}{K(x, y, z)} u-$

$\frac{C_{F} \rho_{n f}}{\sqrt{K(x, y, z)}} \sqrt{u^{2}+v^{2}+w^{2}} u-\sigma_{n f} B_{0}^{2} \frac{u}{\varepsilon}+\frac{\rho_{p}}{\tau_{v}}\left(u_{p}-u\right)$

$\rho_{n f}\left[\frac{1}{\varepsilon} \frac{\partial v}{\partial t}+\frac{u}{\varepsilon^{2}} \frac{\partial v}{\partial x}+\frac{v}{\varepsilon^{2}} \frac{\partial v}{\partial y}+\frac{w}{\varepsilon^{2}} \frac{\partial v}{\partial z}\right]=-\frac{\partial p}{\partial y}+\frac{\mu_{n f}}{\varepsilon}\left(\frac{\partial^{2} v}{\partial x^{2}}+\frac{\partial^{2} v}{\partial y^{2}}+\frac{\partial^{2} v}{\partial z}\right)-\frac{\mu_{n f}}{K(x, y, z)} v-$ $\frac{C_{F} \rho_{n f}}{\sqrt{K(x, y, z)}} \sqrt{u^{2}+v^{2}+w^{2}} v-\sigma_{n f} B_{0}^{2} \frac{v}{\varepsilon}+\frac{\rho_{p}}{\tau_{v}}\left(v_{p}-v\right)$

$\rho_{n f}\left[\frac{1}{\varepsilon} \frac{\partial w}{\partial t}+\frac{u}{\varepsilon^{2}} \frac{\partial w}{\partial x}+\frac{v}{\varepsilon^{2}} \frac{\partial w}{\partial y}+\frac{w}{\varepsilon^{2}} \frac{\partial w}{\partial z}\right]=-\frac{\partial p}{\partial z}+\frac{\mu_{n f}}{\varepsilon}\left(\frac{\partial^{2} w}{\partial x^{2}}+\frac{\partial^{2} w}{\partial y^{2}}+\frac{\partial^{2} w}{\partial z}\right)-\frac{\mu_{n f}}{K(x, y, z)} w-$

$\frac{C_{F} \rho_{n f}}{\sqrt{K(x, y, z)}} \sqrt{u^{2}+v^{2}+w^{2}} w+(\rho \beta)_{n f} g\left(T-T_{c}\right)+\frac{\rho_{p}}{\tau_{v}}\left(w_{p}-w\right)$ 


$$
\begin{aligned}
& {\left[(1-\varepsilon)\left(\rho c_{p}\right)_{p}+\varepsilon\left(\rho c_{p}\right)_{n f}\right] \frac{\partial T}{\partial t}+\left(\rho C_{p}\right)_{n f}\left(u \frac{\partial T}{\partial x}+v \frac{\partial T}{\partial y}+w \frac{\partial T}{\partial z}\right)=} \\
& \frac{\partial}{\partial x}\left(k_{e f f}(x, y, z) \frac{\partial T}{\partial x}\right) \frac{\partial}{\partial y}\left(k_{e f f}(x, y, z) \frac{\partial T}{y}\right)+\frac{\partial}{\partial z}\left(k_{e f f}(x, y, z) \frac{\partial T}{\partial z}\right)+\varepsilon\left(\rho C_{p}\right)_{p}\left[D _ { B } \left(\frac{\partial \phi}{\partial x} \frac{\partial T}{\partial x}+\right.\right. \\
& \left.\left.\frac{\partial \phi}{\partial y} \frac{\partial T}{\partial y}+\frac{\partial \phi}{\partial z} \frac{\partial T}{\partial z}\right)+\frac{D_{T}}{T}\left(\left(\frac{\partial T}{\partial x}\right)^{2}+\left(\frac{\partial T}{\partial y}\right)^{2}+\left(\frac{\partial T}{\partial z}\right)^{2}\right)\right]+\frac{\rho_{p} c_{s}}{\tau_{T}}\left(T_{p}-T\right) \\
& \left(\frac{\partial \varphi}{\partial t}+\frac{u}{\varepsilon} \frac{\partial \varphi}{\partial x}+\frac{v}{\varepsilon} \frac{\partial \varphi}{\partial y}+\frac{w}{\varepsilon} \frac{\partial \varphi}{\partial z}\right)=\frac{\partial}{\partial x}\left(D_{B} \frac{\partial \varphi}{\partial x}\right)+\frac{\partial}{\partial y}\left(D_{B} \frac{\partial \varphi}{\partial y}\right)+\frac{\partial}{\partial z}\left(D_{B} \frac{\partial \varphi}{\partial z}\right)+\frac{\partial}{\partial x}\left(\frac{D_{T}}{T} \frac{\partial T}{\partial x}\right)+ \\
& \frac{\partial}{\partial y}\left(\frac{D_{T}}{T} \frac{\partial T}{\partial y}\right)+\frac{\partial}{\partial z}\left(\frac{D_{T}}{T} \frac{\partial T}{\partial z}\right),
\end{aligned}
$$

\section{Dusty phase}

$\frac{\partial u_{p}}{\partial x}+\frac{\partial v_{p}}{\partial y}+\frac{\partial w_{p}}{\partial z}=0$

$\rho_{p}\left[\frac{\partial U_{p}}{\partial \tau}+U_{p} \frac{\partial U_{p}}{\partial X}+V_{p} \frac{\partial U_{p}}{\partial Y}+W_{p} \frac{\partial U_{p}}{\partial Z}\right]=-\frac{\partial p_{p}}{\partial x}-\frac{\rho_{p}}{\tau_{v}}\left(u_{p}-u\right)$

$\rho_{p}\left[\frac{\partial v_{p}}{\partial t}+u_{p} \frac{\partial v_{p}}{\partial x}+v_{p} \frac{\partial v_{p}}{\partial y}+w_{p} \frac{\partial v_{p}}{\partial z}\right]=-\frac{\partial p_{p}}{\partial y}-\frac{\rho_{p}}{\tau_{v}}\left(v_{p}-v\right)$

$\rho_{p}\left[\frac{\partial w_{p}}{\partial t}+u_{p} \frac{\partial w_{p}}{\partial x}+v_{p} \frac{\partial w_{p}}{\partial y}+w_{p} \frac{\partial w_{p}}{\partial z}\right]=-\frac{\partial p_{p}}{\partial z}-\frac{\rho_{p}}{\tau_{v}}\left(w_{p}-w\right)$

$\rho_{p} c_{s}\left[\frac{\partial T_{p}}{\partial t}+u_{p} \frac{\partial T_{p}}{\partial x}+v_{p} \frac{\partial T_{p}}{\partial y}+w_{p} \frac{\partial T_{p}}{\partial z}\right]=-\frac{\rho_{p} c_{s}}{\tau_{T}}\left(T_{p}-T\right)$

Also, the heterogeneity of the porous medium is represented as:

$K(x, y, z)=K_{0} e^{\eta_{1} x+\eta_{2} y+\eta_{3} z}$

$k_{e f f}(x, y, z)=(1-\varepsilon) k_{p}(x, y, z)+\varepsilon k_{n f}=(1-\varepsilon) k_{0} e^{\eta_{1} x+\eta_{2} y+\eta_{3} z}+\varepsilon k_{n f}$

where the permeability and thermal conductivity of the homogeneous case are $K_{0}$ and $k_{0}$, respectively and the rates of changing of $\ln K$ in the three dimensional are $\eta_{1}, \eta_{2}, \eta_{3}$.

Using the following dimensionless parameters:

$$
\begin{aligned}
& X=\frac{x}{L}, Y=\frac{y}{L}, Z=\frac{z}{L} \tau=\frac{t v_{f}}{L^{2}}, U=\frac{u L}{v_{f}}, V=\frac{v L}{v_{f}}, W=\frac{w L}{v_{f}}, \alpha_{f}=\frac{k_{f}}{\left(\rho C_{P}\right)_{f}}, U_{P}=\frac{u_{P} L}{v_{f}}, V_{P}= \\
& \frac{v_{P} L}{v_{f}}, W_{P}=\frac{w_{p L}}{v_{f}}, P=\frac{p L^{2}}{\rho_{n f} v_{f}^{2}}, P_{P}=\frac{P_{P} L^{2}}{\rho_{n f} v_{f}{ }^{2}}, \theta=\frac{T-T_{c}}{T_{h}-T_{c}}, \theta_{p}=\frac{T_{p}-T_{c}}{T_{h}-T_{c}} \\
& \varphi^{*}=\frac{\varphi}{\varphi_{a v g}}, D_{B}^{*}=\frac{D_{B}}{D_{B o}}, D_{T}^{*}=\frac{D_{T}}{D_{T o}}, \delta=\frac{T_{c}}{T_{h}-T_{c}}
\end{aligned}
$$


Applying Eq. (14), the following dimensionless systems are obtained:

\section{Nanofluids phase:}

$$
\begin{aligned}
& \frac{\partial U}{\partial X}+\frac{\partial V}{\partial Y}+\frac{\partial W}{\partial Z}=0 \\
& \frac{\rho_{\mathrm{nf}}}{\rho_{f}}\left[\frac{1}{\varepsilon} \frac{\partial U}{\partial \tau}+\frac{U}{\varepsilon^{2}} \frac{\partial U}{\partial X}+\frac{V}{\varepsilon^{2}} \frac{\partial U}{\partial Y}+\frac{W}{\varepsilon^{2}} \frac{\partial U}{\partial Z}\right]=-\frac{\rho_{\mathrm{nf}}}{\rho_{f}} \frac{\partial P}{\partial X}+\frac{1}{\varepsilon} \frac{\mu_{n f}}{\mu_{f}}\left(\frac{\partial^{2} U}{\partial X^{2}}+\frac{\partial^{2} U}{\partial Y^{2}}+\frac{\partial^{2} U}{\partial Z^{2}}\right)-\frac{\mu_{n f}}{\mu_{f} K^{*}(X, Y, Z) D a} U- \\
& \frac{\rho_{n f}}{\rho_{f}} \frac{C_{F}}{\sqrt{D a K^{*}(X, Y, Z)}} \sqrt{U^{2}+V^{2}+W^{2}} U-\frac{\sigma_{n f}}{\sigma_{f}} H a^{2} \frac{U}{\varepsilon}+\alpha_{d} D_{s}\left(U_{p}-U\right) \\
& \frac{\rho_{\mathrm{nf}}}{\rho_{f}}\left[\frac{1}{\varepsilon} \frac{\partial V}{\partial \tau}+\frac{U}{\varepsilon^{2}} \frac{\partial V}{\partial X}+\frac{V}{\varepsilon^{2}} \frac{\partial V}{\partial Y}+\frac{W}{\varepsilon^{2}} \frac{\partial V}{\partial Z}\right]=-\frac{\rho_{\mathrm{nf}}}{\rho_{f}} \frac{\partial P}{\partial Y}+\frac{1}{\varepsilon} \frac{\mu_{n f}}{\mu_{f}}\left(\frac{\partial^{2} V}{\partial X^{2}}+\frac{\partial^{2} V}{\partial Y^{2}}+\frac{\partial^{2} V}{\partial Z^{2}}\right)-\frac{\mu_{n f}}{\mu_{f} K^{*}(X, Y, Z) D a} V- \\
& \frac{\rho_{n f}}{\rho_{f}} \frac{C_{F}}{\sqrt{D a K^{*}(X, Y, Z)}} \sqrt{U^{2}+V^{2}+W^{2}} U-\frac{\sigma_{n f}}{\sigma_{f}} H a^{2} \frac{V}{\varepsilon}+\alpha_{d} D_{s}\left(V_{p}-V\right) \\
& \frac{\rho_{\mathrm{nf}}}{\rho_{f}}\left[\frac{1}{\varepsilon} \frac{\partial W}{\partial \tau}+\frac{U}{\varepsilon^{2}} \frac{\partial W}{\partial X}+\frac{V}{\varepsilon^{2}} \frac{\partial W}{\partial Y}+\frac{W}{\varepsilon^{2}} \frac{\partial W}{\partial Z}\right]=-\frac{\rho_{\mathrm{nf}}}{\rho_{f}} \frac{\partial P}{\partial Z}+\frac{1}{\varepsilon} \frac{\mu_{n f}}{\mu_{f}}\left(\frac{\partial^{2} W}{\partial X^{2}}+\frac{\partial^{2} W}{\partial Y^{2}}+\frac{\partial^{2} W}{\partial Z^{2}}\right)- \\
& \frac{\mu_{n f}}{\mu_{f} K^{*}(X, Y, Z) D a} W-\frac{\rho_{n f}}{\rho_{f}} \frac{C_{F}}{\sqrt{D a K^{*}(X, Y, Z)}} \sqrt{U^{2}+V^{2}+W^{2}} W+\frac{(\rho \beta)_{n f}}{(\rho \beta)_{f}} \frac{R a}{P r} \theta+\alpha_{d} D_{s}\left(W_{p}-W\right)
\end{aligned}
$$

$$
\begin{aligned}
& \gamma \frac{\partial \theta}{\partial \tau}+U \frac{\partial \theta}{\partial X}+V \frac{\partial \theta}{\partial Y}+W \frac{\partial \theta}{\partial Z}=\frac{\left(\rho c_{p}\right)_{f}}{\left(\rho c_{p}\right)_{n f}} \frac{1}{P r}\left[\frac{\partial}{\partial X}\left(\xi(X, Y, Z) \frac{\partial \theta}{\partial X}\right)+\frac{\partial}{\partial Y}\left(\xi(X, Y, Z) \frac{\partial \theta}{\partial Y}\right)+\right. \\
& \left.\frac{\partial}{\partial Z}\left(\xi(X, Y, Z) \frac{\partial \theta}{\partial Z}\right)\right]+\frac{\varepsilon\left(\rho c_{p}\right)_{f}}{\left(\rho c_{p}\right)_{n f}} \frac{1}{L e P r}\left[D_{B}^{*}\left[\frac{\partial \varphi^{*}}{\partial X} \frac{\partial \theta}{\partial X}+\frac{\partial \varphi^{*}}{\partial Y} \frac{\partial \theta}{\partial Y}+\frac{\partial \varphi^{*}}{\partial Z} \frac{\partial \theta}{\partial Z}\right]+\frac{D_{T}^{*}}{N_{B T}} \frac{\left[\left(\frac{\partial \theta}{\partial X}\right)^{2}+\left(\frac{\partial \theta}{\partial Y}\right)^{2}+\left(\frac{\partial \theta}{\partial Z}\right)^{2}\right]}{1+\frac{\theta}{\delta}}\right]+ \\
& \frac{2}{3 P r} \frac{\left(\rho C_{p}\right)_{f}}{\left(\rho C_{p}\right)_{n f}} D_{S} \alpha_{d}\left(\theta_{p}-\theta\right) \\
& {\left[\frac{\partial \varphi^{*}}{\partial \tau}+\frac{U}{\varepsilon} \frac{\partial \varphi^{*}}{\partial X}+\frac{V}{\varepsilon} \frac{\partial \varphi^{*}}{\partial Y}+\frac{W}{\varepsilon} \frac{\partial \varphi^{*}}{\partial Z}\right]=\frac{1}{S c} \frac{\partial}{\partial X}\left(D_{B}^{*} \frac{\partial \varphi^{*}}{\partial X}\right)+\frac{1}{S c} \frac{\partial}{\partial Y}\left(D_{B}^{*} \frac{\partial \varphi^{*}}{\partial Y}\right)+\frac{1}{S c} \frac{\partial}{\partial Z}\left(D_{B}^{*} \frac{\partial \varphi^{*}}{\partial Z}\right)+} \\
& \frac{1}{S c} \frac{\partial}{\partial X}\left(\frac{D_{T}^{*}}{N_{B T}\left(1+\frac{\theta}{\delta}\right)} \frac{\partial \theta}{\partial X}\right)+\frac{1}{S c} \frac{\partial}{\partial Y}\left(\frac{D_{T}^{*}}{N_{B T}\left(1+\frac{\theta}{\delta}\right)} \frac{\partial \theta}{\partial Y}\right)+\frac{1}{S c} \frac{\partial}{\partial Z}\left(\frac{D_{T}^{*}}{N_{B T}\left(1+\frac{\theta}{\delta}\right)} \frac{\partial \theta}{\partial Z}\right)
\end{aligned}
$$

\section{Dusty phase:}

$$
\begin{aligned}
& \frac{\partial U_{p}}{\partial X}+\frac{\partial V_{p}}{\partial Y}+\frac{\partial W_{p}}{\partial Z}=0 \\
& {\left[\frac{\partial u_{p}}{\partial t}+u_{p} \frac{\partial u_{p}}{\partial x}+v_{p} \frac{\partial u_{p}}{\partial y}+w_{p} \frac{\partial u_{p}}{\partial z}\right]=-\frac{\rho_{n f}}{\rho_{p}} \frac{\partial P_{p}}{\partial X}-\alpha_{d}\left(U_{p}-U\right)} \\
& {\left[\frac{\partial V_{p}}{\partial \tau}+U_{p} \frac{\partial V_{p}}{\partial X}+V_{p} \frac{\partial V_{p}}{\partial Y}+W_{p} \frac{\partial V_{p}}{\partial Z}\right]=-\frac{\rho_{n f}}{\rho_{p}} \frac{\partial P_{p}}{\partial Y}-\alpha_{d}\left(V_{p}-V\right)}
\end{aligned}
$$


$\left[\frac{\partial W_{p}}{\partial \tau}+U_{p} \frac{\partial W_{p}}{\partial X}+V_{p} \frac{\partial W_{p}}{\partial Y}+W_{p} \frac{\partial W_{p}}{\partial Z}\right]=-\frac{\rho_{n f}}{\rho_{p}} \frac{\partial P_{p}}{\partial Z}-\alpha_{d}\left(W_{p}-W\right)$

$\frac{\partial \theta_{p}}{\partial \tau}+U_{p} \frac{\partial \theta_{p}}{\partial X}+X_{p} \frac{\partial \theta_{p}}{\partial Y}+W \frac{\partial \theta_{p}}{\partial Z}=-\frac{2}{3} \frac{\alpha_{d}}{\omega P r}\left(\theta_{p}-\theta\right)$

$K^{*}(X, Y, Z)=\frac{K(x, y, z)}{K_{0}}=e^{\eta_{1} L X+\eta_{2} L Y+\eta_{3} L Z}$

$\xi(X, Y, Z)=(1-\varepsilon) e^{\eta_{1} L X+\eta_{2} L Y+\eta_{3} L Z}+\varepsilon \frac{k_{n f}}{k_{0}}$

$\gamma=\frac{(1-\varepsilon)\left(\rho c_{p}\right)_{p}+\varepsilon\left(\rho c_{p}\right)_{n f}}{\left(\rho c_{p}\right)_{n f}}$

where,

$R a=\frac{g \beta_{f}\left(T_{h}-T_{c}\right) L^{3}}{\alpha_{f} v_{f}}, \operatorname{Pr}=\frac{v_{f}}{\alpha_{f}}, H a^{2}=\frac{\sigma_{f} L^{2} B_{0}^{2}}{\mu_{f}}, \alpha_{f}=\frac{k_{0}}{\left(\rho c_{p}\right)_{f}}, D a=\frac{K_{0}}{L^{2}}, S c=\frac{v_{\mathrm{f}}}{D_{B 0}}, L e=$

$\frac{k_{o}}{\left(\rho C_{P}\right)_{p} \varphi_{a v g} D_{B 0}}, N_{B T}=\frac{\varphi_{a v g} D_{B 0} T_{c}}{D_{T 0}\left(T_{h}-T_{c}\right)}, D_{T o}=\gamma \frac{\mu_{f}}{\rho_{f}} \varphi_{a v g}, D_{B 0}=\frac{K_{B} T_{c}}{3 \pi \mu_{f} d_{p}}, D_{s}=\frac{\rho_{p}}{\rho_{f}}, \alpha_{d}=\frac{L^{2}}{v_{f} \tau_{v}}$,

$\omega=\frac{c_{s}}{c_{p}}, \tau_{t}=\frac{3}{2} \tau_{v} \omega \operatorname{Pr}$

The boundary conditions can be written as:

$X=1: U=V=W=U_{p}=V_{p}=W_{p}=0, \quad \theta=\theta_{p}=0, \nabla \varphi^{*} \cdot n=-\frac{D_{T}^{*}}{D_{B}^{*}} \frac{1}{\left(1+\frac{\theta}{\delta}\right) N_{B T}} \nabla \theta \cdot n$

$Y=0:=V=W=U_{p}=V_{p}=W_{p}=0, \frac{\partial \theta}{\partial Y}=\frac{\partial \theta_{p}}{\partial Y}=0, \frac{\partial \varphi}{\partial Y}=0$,

$Y=1:=V=W=U_{p}=V_{p}=W_{p}=0, \frac{\partial \theta}{\partial Y}=\frac{\partial \theta_{p}}{\partial Y}=0, \frac{\partial \varphi}{\partial Y}=0$,

$Z=0:=V=W=U_{p}=V_{p}=W_{p}=0, \theta=\theta_{p}=0, \nabla \varphi^{*} \cdot n=-\frac{D_{T}^{*}}{D_{B}^{*}} \frac{1}{\left(1+\frac{\theta}{\delta}\right) N_{B T}} \nabla \theta \cdot n$

$Z=1:=V=W=U_{p}=V_{p}=W_{p}=0 \quad \theta=\theta_{p}=0, \nabla \varphi^{*} . n=-\frac{D_{T}^{*}}{D_{B}^{*}} \frac{1}{\left(1+\frac{\theta}{\delta}\right) N_{B T}} \nabla \theta . n$

On the of solid cylinder (hot) , $\theta=\theta_{p}=1, \nabla \varphi^{*} . n=-\frac{D_{T}^{*}}{D_{B}^{*}} \frac{1}{\left(1+\frac{\theta}{\delta}\right) N_{B T}} \nabla \theta \cdot n$

On the of solid cylinder (cold), $\theta=\theta_{p}=0, \nabla \varphi^{*} . n=-\frac{D_{T}^{*}}{D_{B}^{*}} \frac{1}{\left(1+\frac{\theta}{\delta}\right) N_{B T}} \nabla \theta \cdot n$ 
The thermophysical properties are given as:

$\frac{\mu_{n f}}{\mu_{f}}=\frac{1}{(1-\phi)^{2.5}}$

$\frac{\rho_{n f}}{\rho_{f}}=(1-\phi)+\phi \frac{\rho_{n p}}{\rho_{f}}$

$\frac{\left(\rho c_{p}\right)_{n f}}{\left(\rho c_{p}\right)_{f}}=(1-\phi)+\phi \frac{\left(\rho c_{p}\right)_{n f}}{\left(\rho c_{p}\right)_{f}}$

$\frac{(\rho \beta)_{n f}}{(\rho \beta)_{f}}=(1-\phi)+\phi \frac{(\rho \beta)_{n p}}{(\rho \beta)_{f}}$

$\frac{\sigma_{n f}}{\sigma_{f}}=1 .+\frac{3(\delta-1) \phi}{(\delta+2)-(\delta-1) \phi}, \delta=\frac{\sigma_{n f}}{\sigma_{f}}$,

Table 1: Values of the thermophysical properties

\begin{tabular}{|c|cccccc|} 
& Material & $\boldsymbol{\rho}$ & $\boldsymbol{\beta} \times \mathbf{1 0}^{-\mathbf{5}}$ & $\boldsymbol{C}_{\boldsymbol{P}}$ & $\boldsymbol{k}$ & $\boldsymbol{\sigma}$ \\
\hline $\mathrm{Al}_{2} \mathrm{O}_{3}$ & Alumina & 3970 & 0.85 & 765 & 40 & $\mathbf{1}$ \\
& & & & & & $\times \mathbf{1 0}^{-\mathbf{1 0}}$ \\
\hline Host fluid & Water [ 250 C] & 997.1 & 21 & 4179 & 0.613 & $\mathbf{0 . 0 5}$ \\
\hline Porous & Glass balls & 2700 & & 840 & 1.05 & \\
matrix & & & & & & \\
& & & & & & \\
\hline
\end{tabular}

Since the porous medium is heterogeneous, then the local $\mathrm{Nu}$ and average $\mathrm{Nu}$ are defined as:

$$
\begin{aligned}
& N u_{l o c}=\left.\frac{\varepsilon k_{n f}+(1-\varepsilon) k_{0} e^{\eta_{1} L X+\eta_{2} L Y+\eta_{3} L Z}}{\varepsilon k_{f}+(1-\varepsilon) k_{0} e^{\eta_{1} L X+\eta_{2} L Y+\eta_{3} L Z}} \frac{\partial \theta}{\partial X}\right|_{X=0} \\
& N u_{a v}=\int_{Z=0}^{1} \int_{Y=0}^{1} N u d Y d Z
\end{aligned}
$$

\section{Numerical method and validation:}

The FVM (finite volume method) with a SIMPLE technique is developed, here, to case of 3D and applied to solve the aforementioned systems of the equation. The details of this methodology are given in Patankar [39] and [40]. The discretization forms of the continuity equations, unsteady, convective, diffusive and source terms are given as:

$\oint_{S} \boldsymbol{V} \cdot \boldsymbol{n} d S=0$ 
$\frac{\rho_{\mathrm{nf}}}{\rho_{f}} \frac{1}{\varepsilon} \frac{\partial}{\partial \tau} \int_{\Omega} \boldsymbol{V} d \Omega+\frac{\rho_{n f}}{\rho_{f}} \frac{C_{F}}{\sqrt{D a K^{*}(X, Y, Z)}} \sqrt{U^{2}+V^{2}+W^{2}} \int_{\Omega} \boldsymbol{V} d \Omega++\alpha_{d} D_{s} \int_{\Omega}\left(\boldsymbol{V}_{p}-\boldsymbol{V}\right) d \Omega+$ $\frac{\rho_{\mathrm{nf}}}{\rho_{f}} \frac{1}{\varepsilon^{2}} \oint_{S}(\boldsymbol{V} \boldsymbol{V}) \cdot \boldsymbol{n} d S+\frac{\rho_{\mathrm{nf}}}{\rho_{f}} \oint_{S} P \cdot n d S-\frac{P r}{\varepsilon} \oint_{S}\left(\frac{\mu_{\mathrm{nf}}}{\mu_{f}} \nabla V\right) \cdot n d S+\frac{\mu_{e f f} P r}{\mu_{n f} K^{*}(X, Y, Z) D a} \int_{\Omega} \boldsymbol{V} d \Omega-$ $\chi \frac{(\rho \beta)_{n f}}{(\rho \beta)_{f}} \operatorname{PrRa} \int_{\Omega} \theta d \Omega=0$

The obtained system is solved iteratively using SUR method with convergence criteria of order $10^{-6}$.

\section{Results and discussion}

In order to understand the physical insight behind this parametric study, a set of graphical illustrations is presented and discussed, here. The distance between the hot/cold cylinders is represented by $\delta$ and its range is taken between 0.3 and 0.6. Also, the range of the Darcy number $D a$, the dusty parameter $\alpha_{d}$ and the average nano-parameter $\phi_{a v}$ are taken, respectively as: $10^{-2} \leq D a \leq 10^{-5}, 0.001 \leq \alpha_{d} \leq 0.1,0.01 \leq \phi_{a v} \leq 0.03$. Furthermore, the heterogeneity of the medium properties is taken in $X-Y$ plane $\eta_{1}=\eta_{2}=1.5, \eta_{3}=0$, in $X-Z$ plane $\eta_{1}=\eta_{3}=1.5, \eta_{2}=0$ and in $Y-Z$ plane $\eta_{2}=\eta_{3}=1.5, \eta_{1}=0$.

Figure 2 displays plots of the temperature distributions, streamlines and dusty velocity $W_{p}$ for the variations of the $\delta$ in case of $\left(\eta_{1}=\eta_{2}=\eta_{3}=1.5\right)$ at $\mathrm{Da}=10^{-3}, \mathrm{Ra}=10^{6}, \mathrm{Ha}=$ $10, \alpha_{d}=0.1, D_{S}=10, \phi_{a v}=0.02$. The results revealed that when $\delta$ is increased, the convective transport is enhanced and as results both of the fluid flow and rate of the heat transfer are augmented. The physical explanation of these behaviors is due to the temperature differences within the flow area; those are increased as the cylinders go far from each other.

Figure 3 shows the plots of the temperature distributions, streamlines and dusty velocity $W_{p}$ for the variations of $\left(\left(\eta_{1}, \eta_{2}, \eta_{3}\right.\right.$ and $\left.D a\right)$ at $R a=10^{6}, H a=10, \alpha_{d}=0.1, D_{S}=10, \phi_{a v}=$ 0.02 . Here, the distance between the cylinder is set as 0.4 . It is noted that the convective situation is weak when the heterogeneity is considered in the $X-Y$ plane $\left(\eta_{1}=\eta_{2}=\right.$ $1.5, \eta_{3}=0, D a=10^{-3}$ ) comparing to the other considered cases. Also, the decrease in the Darcy number $\left(\eta_{2}=\eta_{3}=1.5, \eta_{1}=0, D a=10^{-4}\right)$ causes a reduction in the flow of the nanofluid and dusty velocities due to the decrease in the permeability of the medium.

Figure 4 illustrates the plots of the dusty temperature distributions and dusty velocity $W_{p}$ for the variations of $\left(\alpha_{d}\right)$ in case of $\left(\eta_{1}=\eta_{2}=\eta_{3}=0\right)$ at $D a=10^{-3}, \delta=0.4, R a=$ 
$10^{6}, H a=10, D_{S}=10, \phi_{a v}=0.02$. The outcomes disclosed that the growing in the dusty parameter $\alpha_{d}$ enhances the dusty temperature gradients and the dusty velocity $W_{p}$. These behaviors returns to the heat exchange between the nanofluid and dusty phases which causes acceleration in the dusty particle velocity. In the same context, Figure 5 depicts the plots of the nanoparticles distributions and streamlines for the variations of the $\phi_{a v}$ in case of $\left(\eta_{1}=\right.$ $\left.\eta_{2}=\eta_{3}=1.5\right) \quad$ at $\quad D a=10^{-3}, R a=10^{6}, \delta=0.4, H a=10, \alpha_{d}=0.1, D_{S}=10, \delta=0.4$. The streamlines shows a lack of response for the variation of the average nanoparticle volume fraction while there are a clear augmentation in the both the distribution and maximum values of the nanoparticles volume fraction as $\phi_{a v}$ is rising.

Figures 6-9 displays the profiles of the average Nusselt coefficient $N u_{a v}$ for the progressing of the time under impacts of the distance between the cylinders, various cases of the heterogeneity of the medium and several values of the Hartmann number Ha. The results revealed that after a while $(\tau \geq 1.5)$, the values of $N u_{a v}$ are stable and there are no any perturbations in their profiles. Additionally, clear enhancements are seen in values of $N u_{a v}$ as $\delta$ and $\mathrm{Ha}$ are rising. The figures, also, disclosed that the cases of the heterogeneity in $X-Y$ and $X-Z$ directions give the lowest values of $N u_{a v}$ due to the decrease in the permeability in these directions.

Figures 10-11 illustrate the profiles of $N u_{a v}$ for the variations of $\alpha_{d}, D a, \delta$ and $\phi_{a v}$ in case of $\left(\eta_{1}=\eta_{2}=\eta_{3}=1.5\right)$ at $R a=10^{6}, \delta=0.4, H a=10, D_{S}=10, \phi_{a v}=0.02$. The dusty coefficient $\alpha_{d}$ has no slightly influences on the values of $N u_{a v}$ while $N u_{a v}$ is augmented, clearly as $D a$ is decreased. Additionally, as stated later, the rising in distance between the cylinders enhances the temperature differences and hence $N u_{a v}$ is growing. Furthermore, the increasing values of $\phi_{a v}$ cause a weakness in the convective situation and hence $N u_{a v}$ is reduced.

\section{Conclusions}

Numerical simulations have been carried out for the 3D magnetic convective transport of dusty nanofluid within $3 \mathrm{D}$ cubic domain filled by porous material. The nanofluid behavior is presented using the non-homogeneous nanofluid model and the non-Darcy model is applied for the flow through the medium. Two-systems of PDE's are presented for the nanofluid and dusty phases and the magnetic influences are taken in Z-direction. As a new addition in this 
type of the flow, the heterogeneity of the medium properties, namely, permeability and thermal conductivity are analyzed. The following major findings can be summarized:

- The three dimensional convective transport of dusty nanofluids can be controlled using two inner isothermal cylinders.

- The growing in the distance between the cylinders enhances the three dimensional dusty flow and the rate of the heat transfer.

- The cases of the heterogeneity in $X-Y$ and $X-Z$ directions have the lowest rate of the heat transfer.

- The rising in Hartmann number enhances the temperature gradients and hence the Nusselt number is augmented.

\section{References}

[1] D.A. Nield, A. Bejan, Convection in Porous Media, 5th ed, Springer, 2017

[2] I. Pop, D.B. Ingham, Convective Heat Transfer, Mathematical and Computational Modeling of Viscous Fluids and Porous Media, Pergamon, Oxford, 2001.

[3] Ingham DB , Pop I . Transport phenomena in porous media III. Elsevier; 2005 . volume 3

[4] A. Bejan, A.D. Kraus (Eds.), Heat Transfer Handbook, Wiley, New York, 2003.

[5] G.V. Kuznetsov, M.A. Sheremet, New approach to the mathematical modeling of thermal regimes for electronic equipment, Russ. Microelectron. 37 (2008) 131-138,

[6] S. Choi, Enhancing thermal conductivity of fluids with nanoparticles, in: D.A. Singer, H.P. Wang (Eds.), Developments and Applications of Nonnewtonian Flows, American Society of Mechanical Engineers, New York, NY, USA, (1995), 99-105.

[7] J. Buongiorno, Convective transport in nanofluids, ASME J. Heat Transf. 128, (2006) $240-250$

[8] X.Q. Wang, Mujumdari A. A review on nanofluids - Part: II experiments and applications. Braz J Chem Eng;25(4), (2008) 631-648..

[9] S..Ozerinc, S.Kakac, A.G.Yazicioglu ,. Enhanced thermal conductivity of nanofluids: a state-of-the-art review, Microfluid Nanofluid8(2),(2010) 145-170. 
[10] M.Chandrasekar, S. Suresh S, A review on the mechanisms of heat transport in nanofluids. Heat Transf Eng30(14) (2009) 1136-1150.

[11] Y.J. Li, J.E. Zhou, S.Tung, E.Schneider, S. Xi, A review on development of nanofluid preparation and characterization, Powder Technol196(2) ( 2009) 89-101

[12] B. Ghasemi, S.M. Aminossadati, A. Raisi, Magnetic field effect on natural convection in a nanofluid-filled square enclosure, Int. J. Therm. Sci. 50 (2011) 1748-1756.)〉

[13] Garoosi F, Bagheri GH, Talebi F. Numerical simulation of natural convection of nanofluids in a square cavity with several pairs of heaters and coolers (HACs) inside. Int $\mathbf{J}$ Heat Mass Transf 2013;67:362-76.

[14]S. E. Ahmed, A. M. Rashad and R.Gorla, Natural convection in triangular enclosures filled with nanofluid saturated porous media, Journal of Thermophysics and Heat Transfer 27(4) (2013) 700-706

[15] M. S. Hossain ,M. Abdul Alim, MHD free convection within trapezoidal cavity with non-uniformly heated bottom wall, International Journal of Heat and Mass Transfer 69 (2014) 327-336

[16] M.A. Sheremeta,b, T. Groşan, , I. Pop, Steady-state free convection in right-angle porous trapezoidal cavity filled by a nanofluid: Buongiorno's mathematical model, European Journal of Mechanics B/Fluids 53 (2015) 241-250

[17] H. Saleh, R. Roslan,I. Hashim, Natural convection heat transfer in a nanofluid-filled trapezoidal enclosure, Int J Heat Mass Transfer 54 (2011)194-201.

[18] Ahmed, SE, Rashed, ZZ: MHD natural convection in a heat generating porous mediumfilled wavy enclosures using Buongiorno's nanofluid model, Case Studies in Thermal Engineering, 14 (2019) 100430.

[19] Z. Z. Rashed, Sameh Elsayed Ahmed, Z. A. S. Raizah ,Thermal dispersion effect on natural convection in inclined rectangular enclosures filled with multi-layers of a heat generating porous medium and nanofluid using Buongiorno's, Journal of Porous Media, DOI: 10.1615/JPorMedia.2020026476, pages 341-361 
[20] I. Jelodari, H. A.. Nikseresht, Effects of Lorentz force and induced electrical field on the thermal performance of a magnetic nanofluid-filled cubic cavity, Journal of Molecular Liquids 252 (2018) 296-310

[21] H. Sajjadi , A. Amiri Delouei, M. Atashafrooz, M. Sheikholeslami, Double MRT Lattice Boltzmann simulation of 3-D MHD natural convection in a cubic cavity with sinusoidal temperature distribution utilizing nanofluid, International Journal of Heat and Mass Transfer 126 (2018) 489-503

[22] L. Wang, B. Shi, Z. Chai, Effects of temperature-dependent properties on natural convection of nanofluids in a partially heated cubic enclosure, Applied Thermal Engineering 128 (2018) 204-213

[23] M. SheikholeslamiS.A. Shehzad, F.M. Abbasi, Zhixiong Lid Nanofluid flow and forced convection heat transfer due to Lorentz forces in a porous lid driven cubic enclosure with hot obstacle, Comput. Methods Appl. Mech. Engrg. 338 (2018) 491-505.

[24] M. A. Sheremet, I. Pop, Marangoni natural convection in a cubical cavity filled with a nanofluid Buongiorno's nanofluid model, Journal of Thermal Analysis and Calorimetry 135 (2019) 357-369.

[25] M. Sheikholeslami, S.A. Shehzad, Z. Li, Water based nanofluid free convection heat transfer in a three dimensional porous cavity with hot sphere obstacle in existence of Lorenz forces, International Journal of Heat and Mass Transfer 125 (2018) 375-386

[26] A. I. Alsabery, M. A. Ismael, A. J. Chamkha , I.Hashim, H. Abulkhair,Unsteady flow and entropy analysis of nanofluids inside cubic porous container holding inserted body and wavy bottom wall, International Journal of Mechanical Sciences 193 (2021) 106161

[27] FE Marble, Dynamics of dusty gases, Ann Rev Fluid Mech, 2( 1970) 397-446

[28] G. Rudinger, Fundamentals of Gas-particle Flow, Elsevier Scientific Publishing Co., Amsterdam, 1980.

[29] S. Naramgari, C. Sulochana, MHD flow of dusty nanofluid over a stretching surface with volume fraction of dust particles, Ain Shams Engineering Journal (2016) 7, 709-716 
[30] N. Begum, S. Siddiqa, M. Sulaiman, S. Islam, M.A. Hossain, R.S.R. Gorla, Numerical solutions for gyrotactic bioconvection of dusty nanofluid along a vertical isothermal surface, International Journal of Heat and Mass Transfer 113 (2017) 229-236.

[31] . S. Siddiqa, N. Begum, M.A. Hossain, R. S R. Gorla, A.A. Al-Rashed ,Two-phase natural convection dusty nanofluid flow, International Journal of Heat and Mass Transfer 118 (2018) 66-74

[ 32 ] B.J. Gireesha, B. Mahanthesh, G.T. Thammanna, P.B. Sampathkumar, Hall effects on dusty nanofluid two-phase transient flow past a stretching sheet using KVL model, Journal of Molecular Liquids 256 (2018) 139-147

[33] S.R. Mishra, M. Ijaz Khan, B.C. Rout, Dynamics of dust particles in a conducting dusty nanomaterials: A computational approach, International Communications in Heat and Mass Transfer 119 (2020) 104967

[34] M. Rashid, · T. Hayat, A. Alsaedi, · B. Ahmed, Flow of Fe3O4 nanofluid with dust and nanoparticles, Applied Nanoscience (2020) 10:3115-3122.

[35] Z. Z. Rashed1 and S. E. Ahmed, Peristaltic Flow of Dusty Nanofluids in Curved Channels, Computers, Materials \& Continua, DOI:10.32604/cmc.2020.012468

[36] Y.J. Zhuang, Q.Y. Zhu, Numerical study on combined buoyancy-Marangoni convection heat and mass transfer of power-law nanofluids in a cubic cavity filled with a heterogeneous porous medium, International Journal of Heat and Fluid Flow 71 (2018) 3954.

[37] Y.J. Zhuang, Q.Y. Zhu, Analysis of entropy generation in combined buoyancyMarangoni convection of power-law nanofluids in 3D heterogeneous porous media, International Journal of Heat and Mass Transfer 118 (2018) 686-707

[38] Z.Z. Rashed, M. Alhazmi, S. E. Ahmed, Non-homogenous nanofluid model for 3D convective flow in enclosures filled with hydrodynamically and thermally heterogeneous porous media, Alexandria Engineering Journal 60(2021) 3119-3132.

[39] S.V. Patankar, Numerical heat transfer and fluid flow. Washington: Hemisphere Pub. Corp, (1980). 
[40] S.E. Ahmed, Mixed convection in thermally anisotropic non-Darcy porous medium in double lid-driven cavity using Bejan's heatlines, Alexandria Engineering Journal, 55 (2016) 299-309. 


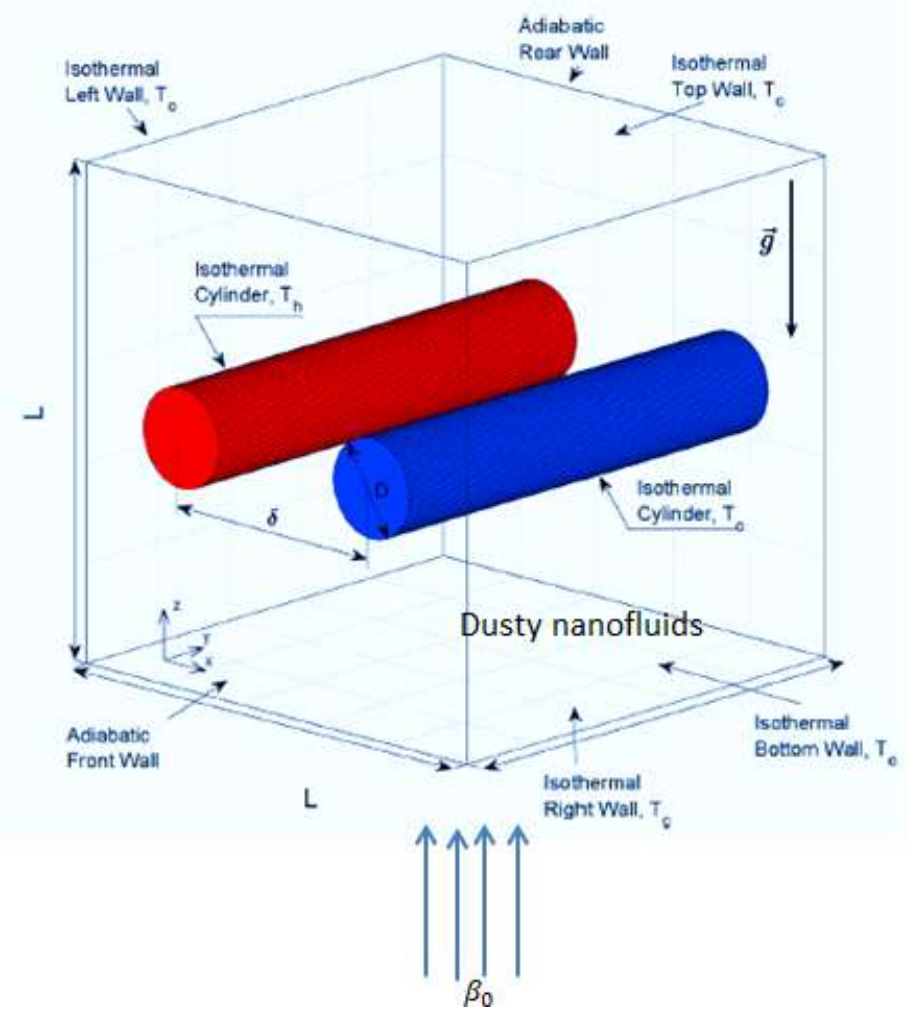

Fig.1. Physical model and problem conditions

$\delta=0.3$

$\delta=0.4$

$\delta=0.5$

$\delta=0.6$ 

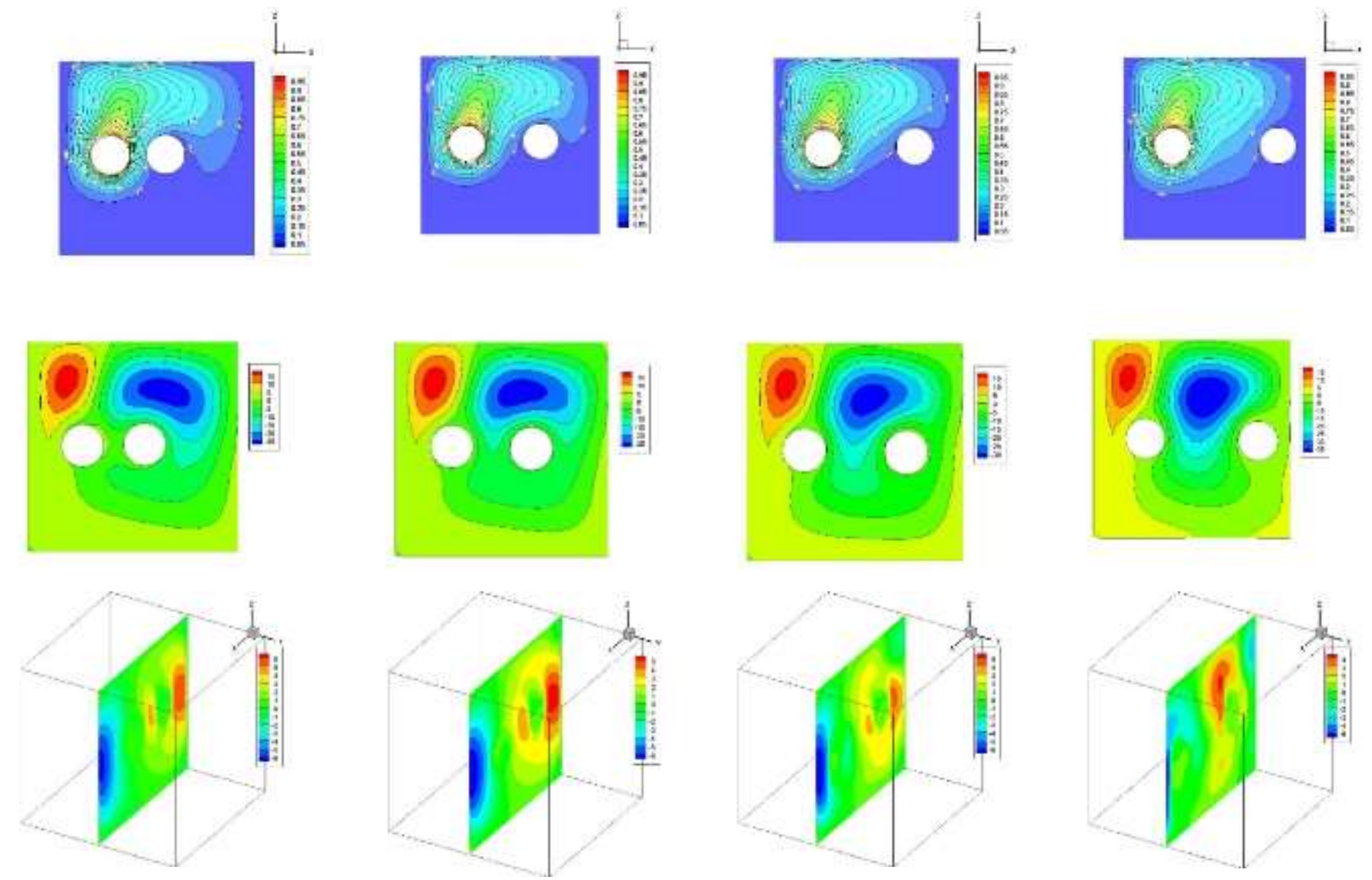

Figure 2: Plots of the temperature distributions, streamlines and dusty velocity $W_{p}$ for the variations of the $\delta$ in case of $\left(\eta_{1}=\eta_{2}=\eta_{3}=1.5\right)$ at $D a=10^{-3}, R a=10^{6}, H a=10, \alpha_{d}=0.1, D_{S}=10, \phi_{a v}=0.02$ 


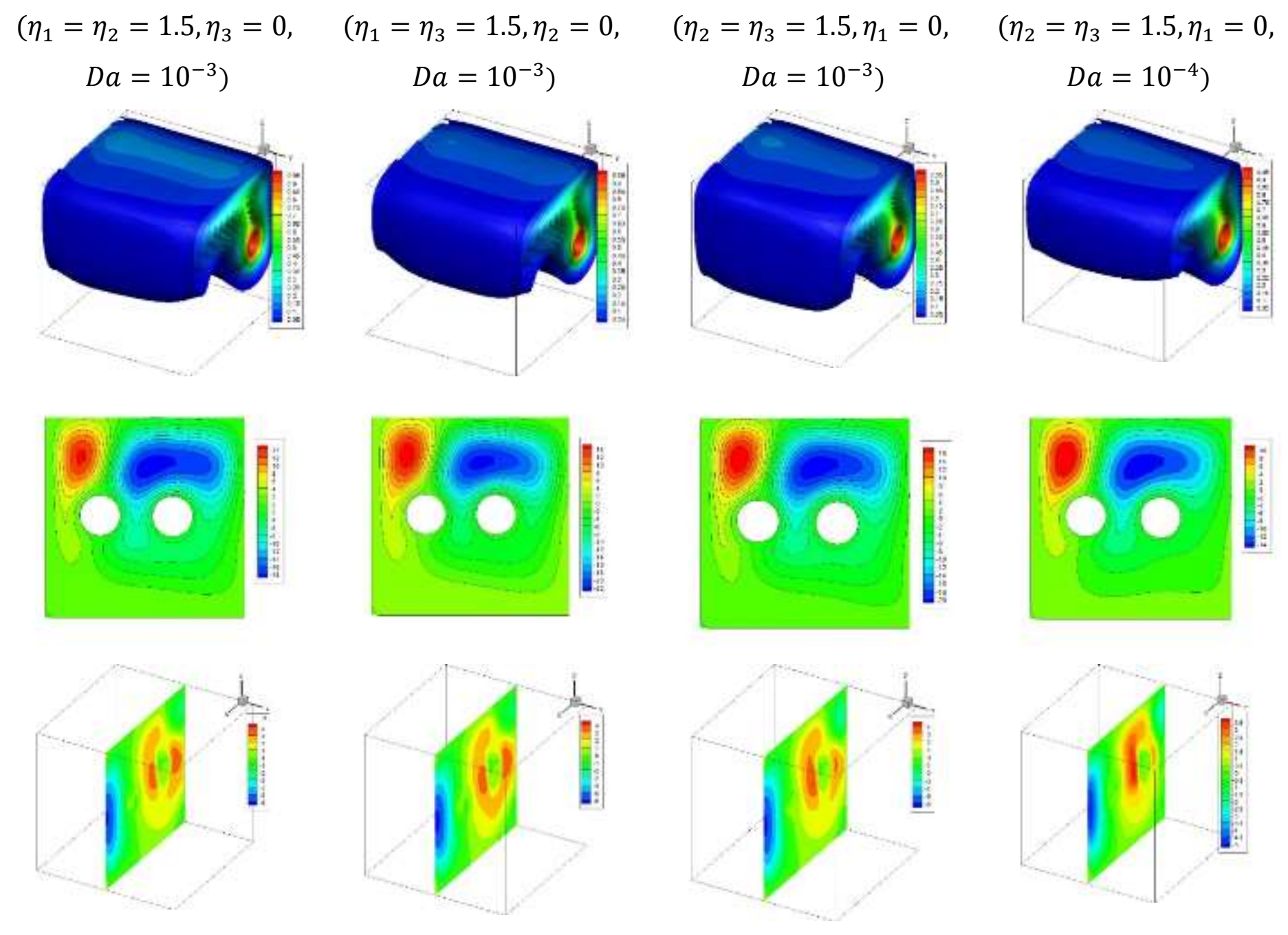

Figure 3: Plots of the temperature distributions, streamlines and dusty velocity $W_{p}$ for the variations of $\left(\left(\eta_{1}, \eta_{2}, \eta_{3}\right.\right.$ and $\left.D a\right)$ at $\delta=0.4, R a=10^{6}, H a=10, \alpha_{d}=0.1, D_{S}=10, \phi_{a v}=0.02$ 

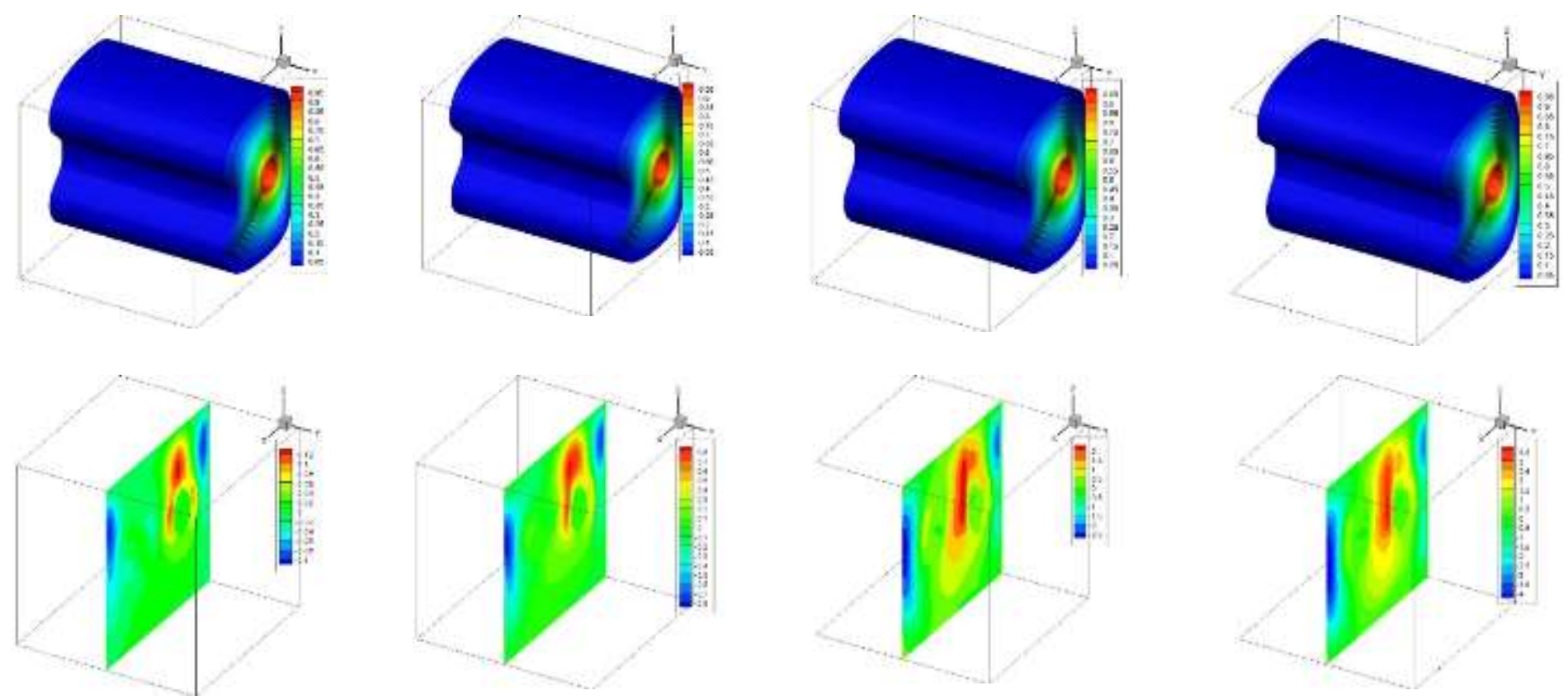

Figure 4: Plots of the dusty temperature distributions and dusty velocity $W_{p}$ for the variations of $\left(\alpha_{d}\right)$ in case of $\left(\eta_{1}=\eta_{2}=\eta_{3}=0\right)$ at $D a=10^{-3}, \delta=0.4, R a=10^{6}, H a=10, D_{S}=10, \phi_{a v}=0.02$ 

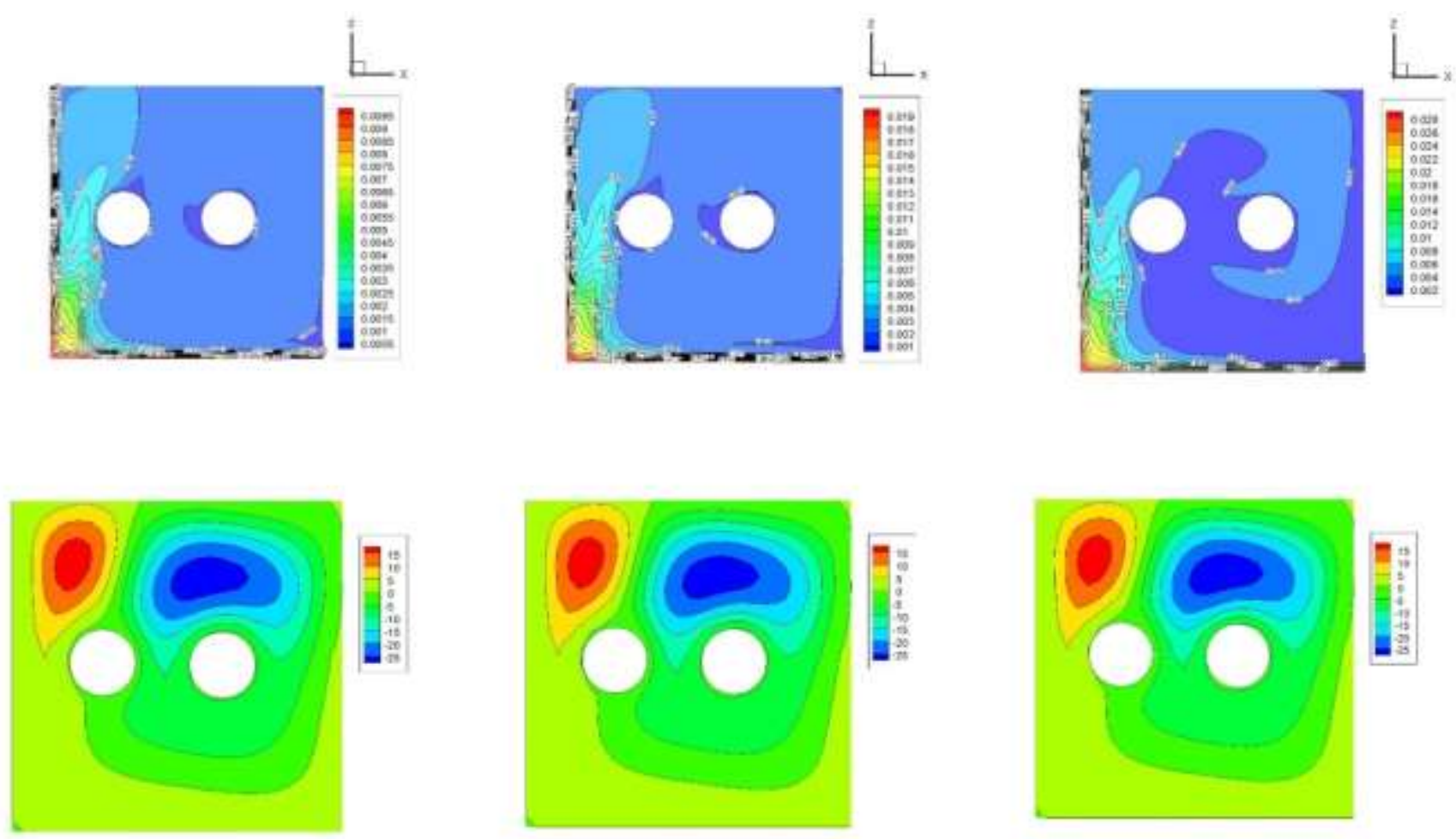

Figure 5: Plots of the nanoparticles distributions and streamlines for the variations of the $\phi_{a v}$ in case of $\left(\eta_{1}=\eta_{2}=\eta_{3}=1.5\right)$ at $\mathrm{Da}=10^{-3}, \mathrm{Ra}=10^{6}, \delta=0.4, \mathrm{Ha}=10, \alpha_{d}=0.1, D_{S}=10, \delta=0.4$ 


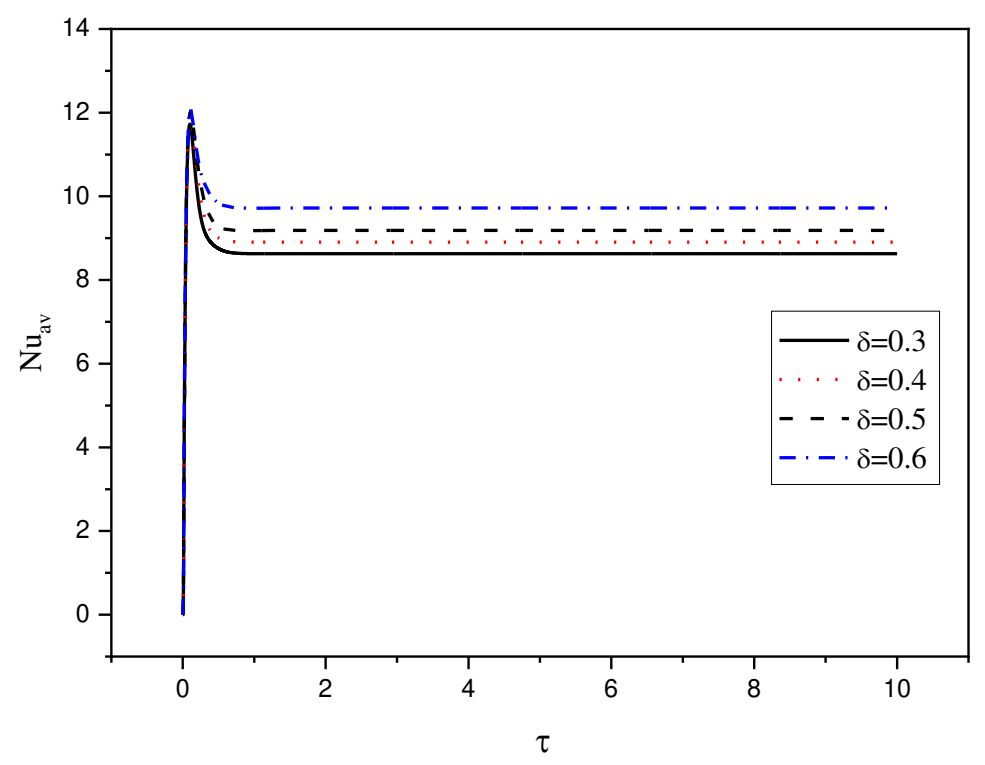

Fig. 6. Profiles of the average Nusselt Number for the variations of $\delta$ in case of $\left(\eta_{1}=\eta_{2}=\right.$ $\left.\eta_{3}=1.5\right)$ at $D a=10^{-3}, R a=10^{6}, H a=10, \alpha_{d}=0.1, D_{S}=10, \phi_{a v}=0.02$

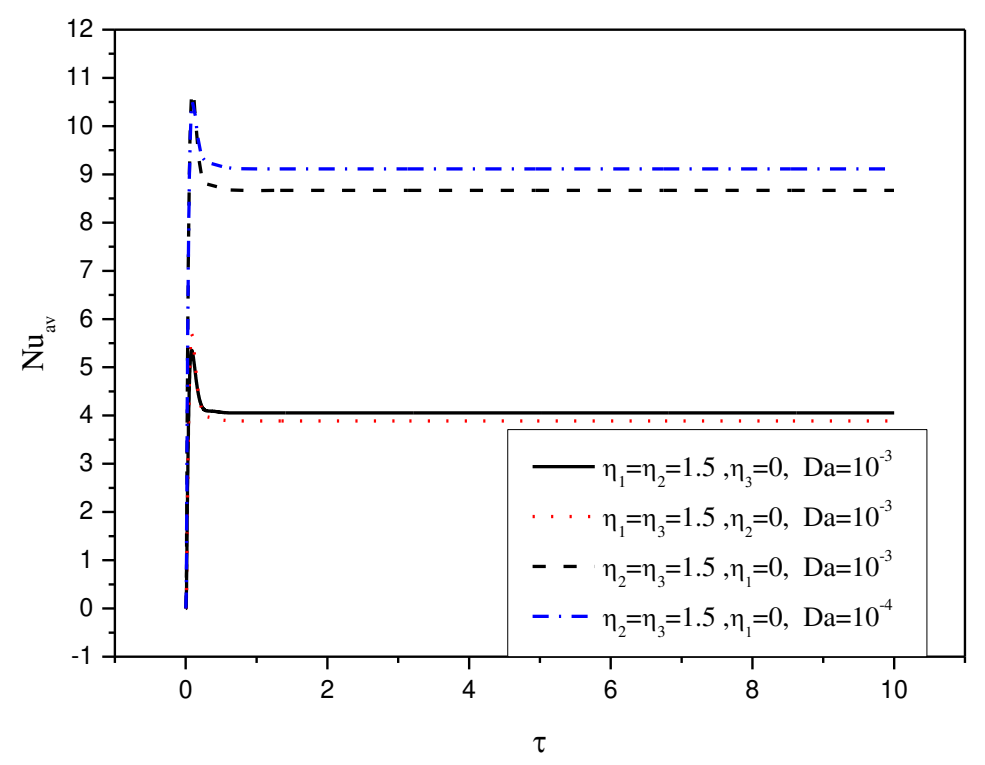

Fig. 7. Profiles of the average Nusselt Number for the variations of $\left(\left(\eta_{1}, \eta_{2}, \eta_{3}\right.\right.$ and $\left.D a\right)$ at $H a=10, R a=10^{6}, H a=10, \alpha_{d}=0.1, D_{S}=10, \delta=0.4, \phi_{a v}=0.02$ 


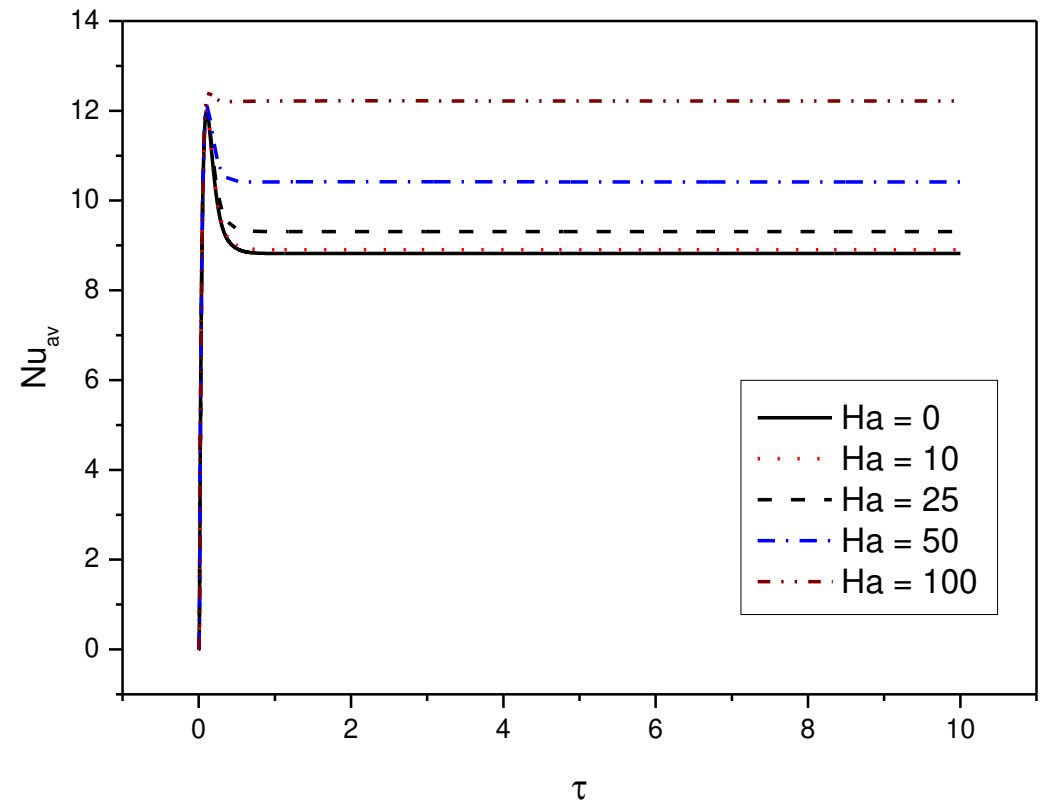

Fig. 8. Profiles of the average Nusselt Number for the variations of $\mathrm{Ha}$ in case of $\left(\eta_{1}=\right.$ $\left.\eta_{2}=\eta_{3}=1.5\right)$ at $D a=10^{-3}, R a=10^{6}, \delta=0.4, \alpha_{d}=0.1, D_{S}=10, \phi_{a v}=0.02$

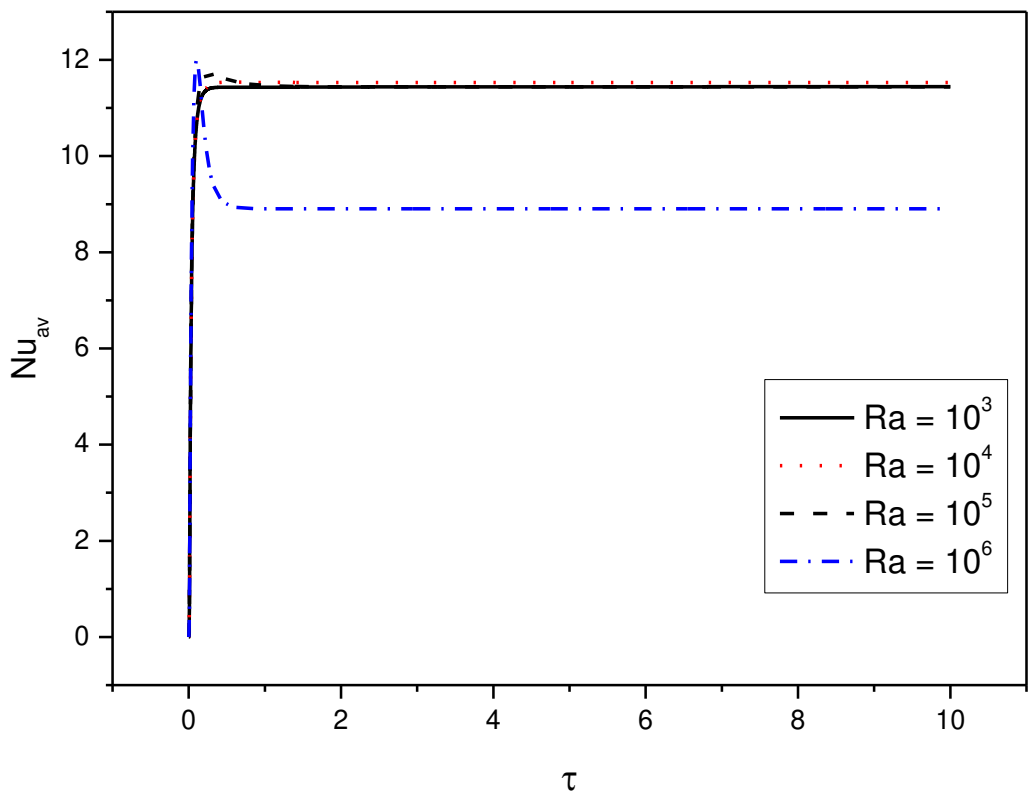

Fig. 9. Profiles of the average Nusselt Number for the variations of $R a$ in case of $\left(\eta_{1}=\right.$ $\left.\eta_{2}=\eta_{3}=1.5\right)$ at $D a=10^{-3}, \delta=0.4, \alpha_{d}=0.1, H a=10, D_{S}=10, \phi_{a v}=0.02$ 


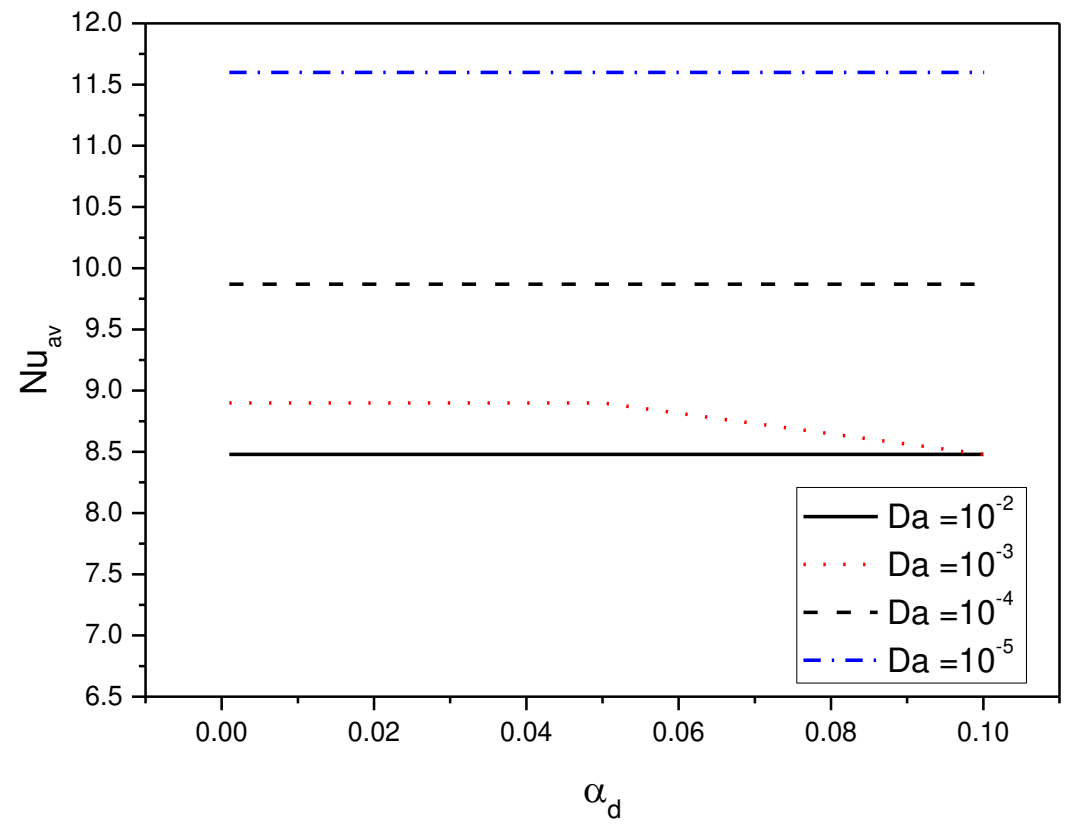

Fig. 10. Profiles of the average Nusselt Number for the variations of $\alpha_{d}$ and $D a$ in case of $\left(\eta_{1}=\eta_{2}=\eta_{3}=1.5\right)$ at $R a=10^{6}, \delta=0.4, H a=10, D_{S}=10, \phi_{a v}=0.02$

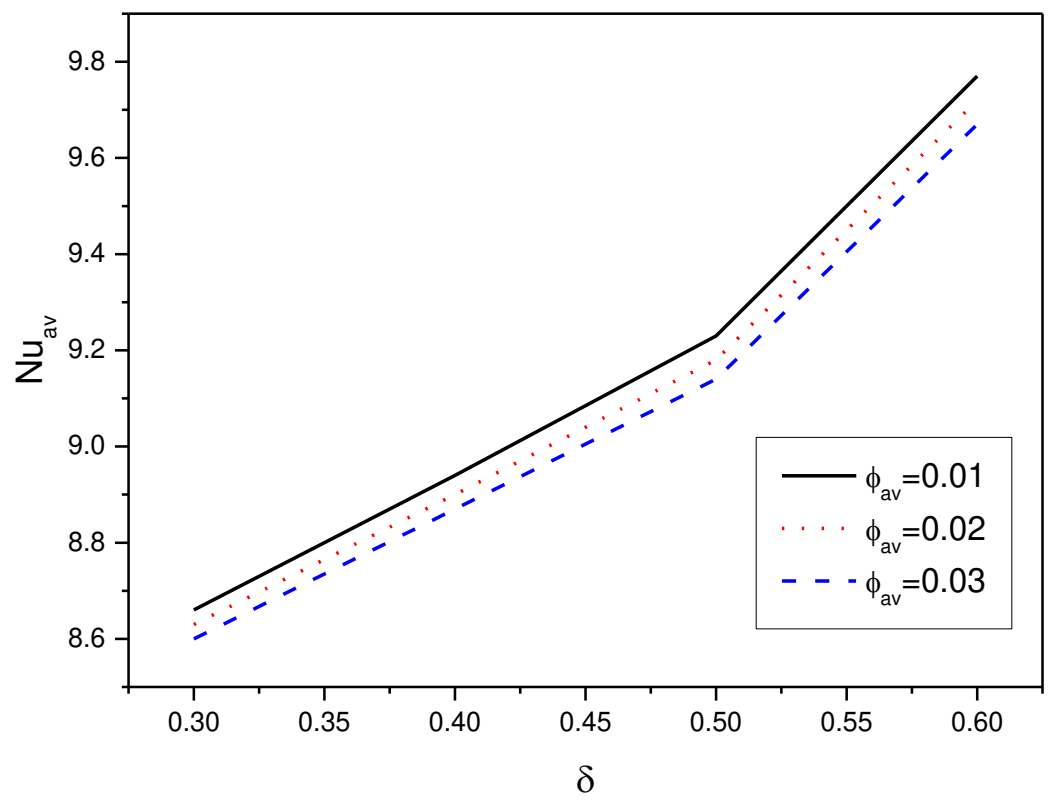

Fig. 11. Profiles of the average Nusselt Number for the variations of $\delta$ and $\phi_{a v}$ in case of $\left(\eta_{1}=\eta_{2}=\eta_{3}=1.5\right)$ at $R a=10^{6}, \mathrm{Da}=10^{-3}, \mathrm{Ha}=10, D_{S}=10, \alpha_{d}=0.1$. 